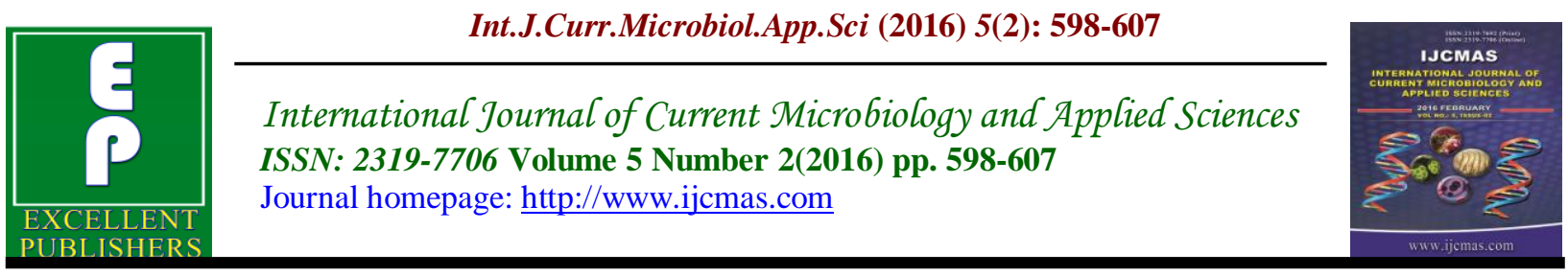

Original Research Article

doi: http://dx.doi.org/10.20546/ijcmas.2016.502.067

\title{
Bacteriological Profile of Septicaemia and Extended Spectrum Beta Lactamases Production in Multi Drug Resistant Strains
}

\section{Introduction}

Blood stream infections (BSIs) are important cause of severe morbidity of patients and ranges from self-limiting infections to life threatening sepsis that require rapid and aggressive antimicrobial treatment. Wasihum et al (2015) BSIs cause by wide spectrum of organisms and this spectrum is subject to geographical alteration. These infections may be polymicrobial or mono-microbial. Common organism isolated were Staphylococcus aureus, Coagulase negative staphylococci, 
Escherichia coli, Pseudomonas spp, Klebsiella spp. Salmonella typhi, Acinetobacter spp, Enterobacter spp. Citrobacter spp, Enterococcus spp, Streptococcus spp, Micrococci and Candida. Usha et al (2007)

Various type of $\beta$ (beta) lactamases increasing worldwide like extended spectrum beta lactamases (ESBL), Amp C $\beta$ lactamases and mettalo-beta lactamases (MBL). Amutha et al (2014) Presence of these enzymes in single isolates reduces the effectiveness of antibiotic chemotherapy. There appears to be a paucity of surveys related to antibiotic resistance from developing countries like Indian subcontinent particularly in this Mewat region.

The main aim of this study was to know common aerobic bacteriological agent of septicaemia and their antibiotic resistance pattern, and emergence of ESBL, Amp C and MBL among multidrug resistant strains.

\section{Duration of study}

A prospective study data was conducted from January 2015 to December 2015 at Research Laboratory of Microbiology Department of the SHKM GMC Mewat, Haryana.

\section{Inclusion criteria}

The study included all clinical isolates obtained from growth of blood culture came to bacteriology lab both OPD as well as IPD, highly suspected of septicaemia.

\section{Exclusion criteria}

1. Patients of HIV and other immunological disorder

2. Patients of diabetes.

3. Patient took antibiotic in last 7 days

\section{Methodology}

$5 \mathrm{ml}$ blood was collected from each adult patient with strict aseptic precaution, and inoculated immediately into $50 \mathrm{ml} \mathrm{BHI}$ broth with $0.025 \%$ of sodium polyanethol sulphonate as anticoagulant (HImedia). In children (below 12 year) $1-2 \mathrm{ml}$ of blood was taken and inoculated in $5-10 \mathrm{ml}$ of BHI broth. The broths were sub cultured on 5\% blood agar and Mac Conkey agar and chocolate agar after overnight incubation. Subculture was repeated at regular interval up to 7 days until the final result was negative. Positive growth was identified by Gram staining, colony characteristics, and standard biochemical tests. Bailey and scott's et al (2007). Antibiotic susceptibility test was put for various antibiotics disc (HiMedia). Ampicillin (10 $\mu \mathrm{g})$, Cefuroxime (30 $\mu \mathrm{g})$, Ceftriaxone $(30 \mu \mathrm{g})$, Ciprofloxacin $(5 \mu \mathrm{g})$, Amoxicillin-Clavulenate $(10 / 20 \mu \mathrm{g})$, Co-trimoxazole $\quad(1.25 / 23.75 \quad \mu \mathrm{g})$, Ceftazidime $(70 \mu \mathrm{g})$, Ceftriaxone $(30 \mu \mathrm{g})$, Gentamicin $(10 \mu \mathrm{g})$, Ciprofloxacin $(5 \mu \mathrm{g})$, Amikacin $(30 \mu \mathrm{g})$, Netilmicin $(30 \mu \mathrm{g})$, Tetracycline $(30 \mu \mathrm{g}), \quad$ Piperacillintazobactam $(100 / 10 \mu \mathrm{g})$ and Imipenem (10 $\mu \mathrm{g})$ (Hi-media, Mumbai). The reference strains used as control for disc diffusion testing were E. coli ATCC 25922, $P$. aeruginosa ATCC 27853, S. aureus ATCC 25923 and E. faecalis ATCC 29212.

The isolate was considered as multidrug resistant (MDR) when non-susceptible to at least one agent in more than three antimicrobial categories/groups and extensively drug resistant (XDR) if nonsusceptible to at least one agent in all but two or fewer antimicrobial categories/ groups i.e. bacterial isolates remain susceptible to only one or two categories. Isolate non-susceptible to all agents in all antimicrobial categories was considered as pan drug-resistant (PDR). Magioracos et al 2012 


\section{ESBL Screening}

All the isolates showing resistance to $3^{\text {rd }}$ generation cephalosporins, namely Ceftazidime, Ceftriaxone and Cefotaxime, were further tested for confirmation of $\beta$ lactamase production by phenotypic methods. CLSI (2014)

\section{Double Disk Synergy Test}

A disc of augmentin (20 $\mu \mathrm{g}$ amoxycillin and $10 \mu \mathrm{g}$ clavulanic acid) and a $30 \mu \mathrm{g}$ disc of ceftazidime was placed $15 \mathrm{~mm}$ out from edge of Augmentine disc at $90^{\circ}$ angle so that its inner edge is $15 \mathrm{~mm}$ from it. Same was performed with Cefotaxime $30 \mu \mathrm{g}$, Ceftriaxone $30 \mu \mathrm{g}$, Aztreonam $30 \mu \mathrm{g}$, Cefpodoxim $10 \mu \mathrm{g}$ so that they were spaced $90^{\circ}$ apart lawn culture of the resistant isolate under test on Mueller-Hinton Agar The zone size around the test antibiotic disc increased towards the Augmentin disc An "enhancement" or extension of the zone of inhibition is seen between any of the cephalosporin antibiotics and the clavulanate containing disks, This phenomenon is often referred to as the "KEYHOLE" effect, or "CLAVULANIC" effect. Gupta et al (2007)

\section{Phenotypic Confirmatory Disk Diffusion Test (PCDDT) for ESBL Detection}

Ceftazidime (30 mcg) was used alone as well as in combination with Clavulanic acid (10mcg). Both the disks were placed on $\mathrm{MH}$ Agar plates pre-swabbed with the respective culture and incubated at $37{ }^{\circ} \mathrm{C}$ for $24 \mathrm{~h}$. An increase in the zone diameter for Ceftazidime-Clavulanic acid by $\geq 5 \mathrm{~mm}$ was considered positive for ESBL production. Jarlier et al (1988)

\section{AmpC Disc Test to Detect Amp C beta Lactamases Production}

A lawn culture of Escherichia coli 25922 was prepared on MHA plate. Sterile disk of $6 \mathrm{~mm}$ were moistened with sterile saline (20 $\mu l)$ and inoculated with several colonies of test organism. The inoculated disk was then placed beside a cefoxitin disk almost touching on the inoculated plate. The plates were incubated at $37^{\circ}$ c overnight. A positive test appeared as flattening or indentation of the Cefoxitin inhibition zone. Black et al (2005)

\section{MRSA and MRCoNS Detection}

Detection of Methicillin resistance among Staphylococcus aureus isolates was done using $1 \mu \mathrm{g}$ oxacillin disc on Mueller Hinton agar supplemented with an additional 5\% $\mathrm{NaCl}$ and Cefoxitin disc (30 ug) diffusion test and results interpreted according to CLSI guidelines. Broekema et al (2009)

\section{Detection of MBL}

MBL production was demonstrated by two methods namely combined disc test with EDTA and Ceftazidime $(30 \mu \mathrm{g})$ - EDTA Double disc synergy test. Marchiaro et al (2005)

\section{Result and Discussion}

Out of 2681 cases studied $2292(85.4 \%)$ were indoor patients while 389 (14.5\%) were from various outpatient departments. Maximum blood samples (59.7\%) were received from Paediatric ward from patients of septicaemia and $11.9 \%$ from Paediatric OPD with PUO, $6.46 \%$ from surgery ward from patients of wound infection and PUO and rest were from various other departments. 1403(52.3\%) were females and $1278(47.7 \%)$ were males. Female were more than male but difference is not significant ( $\mathrm{p}$ value $<0.05$ ) Age of patients was ranged from neonates to elderly ( 0 to 86 years). Maximum patients were from neonatal ICU, mean age of patients were 
15.1years. Growth was obtained in 571 samples out of 2681 (21.56\%) in which 96\% showed bacterial growth and Candida spp was isolated from $4 \%$ of the samples. In most of samples there was mono-microbial growth only in $3.25 \%$ of cases two or more microorganisms were isolated. In our study $21.1 \%$ of Enterobacteriaceae produced ESBL, $6.4 \%$ produced Amp C $\beta$ lactamase and $6.3 \%$ produced both type of $\beta$ lactamases. Two isolates of pseudomonas were MBL positive. 23\% organism were multi drug resistant (MDR) in all isolates. There was no PDR found in our study. In gram positive cocci $53 \%$ were MRSA and $43 \%$ were MRCoNS.

The results of our study demonstrate the distribution of microbial isolates causing septicaemia and their susceptibility pattern to most commonly used oral and parenteral antimicrobial agents.

In most cases of septicaemia, a single microorganism was isolated from blood, while in $3.25 \%$ of cases two or more microorganisms were isolated. The polymicrobial blood stream infections have been reported by various workers with an incidence ranging from $4.7-18.7 \%$, most of which were hospital acquired. Garg et al (2007), Usha et al (2007), Kalpesh et al (2014). In our study blood culture positivity rate was $21.29 \%$ that was similar to other study held in India by Garg et al (2007)(20.1\%) however it was ranged from $8 \%$ to $10 \%$ by other investigators. Maximum patients were from paediatric age group from new born to 14 years. Manjula et al (2005), Vanitha et al (2012), Sumita et al (2014)

Table No. 1 shows maximum isolates were pseudomonas spp followed by klebsiellae spp and staphylococci aureus. These organisms can survive in the environment for a relatively long time and widely distributed in the hospital environment and have high risk for being transmitted from the environment to the patients through practices that breach infection control measures. Anantham et al (2005). This emphasises the need for the establishment of effective and functional infection control programmes in hospitals. Dechen et al (2009). Antibiotics used for susceptibility testing for Gram-negative isolates, Ciprofloxacin, Cefotaxime, Gentamycin and Amoxycillin / Clavulanate was good effective against Enterobacteriaceae, whereas for non-fermenters like Pseudomonas spp. and Acinetobacter spp. Ofloxacin and Amikacin was more active. However, the combination of Piperacillintazobactam and Imipenem put up for all Gram-negative isolates showed the highest activity among all antibiotics used for these isolates. Our findings were similar to other investigators. Usha et al (2007) Vinod et al (2011) (Table No. 3) In gram positive cocci maximum isolates were $S$. aureus which was found to be less susceptible to Ampicillin, Erythromycin, and Clindamycin. Other antibiotics like Cotrimoxazol, Ciprofloxacin, Gentamycin, Amoxycillin/Clavulenate have good activity against all gram positive cocci and none of the strains showed resistance to Vancomycin and Teicoplanin. (Table No. 2) They could be used in multidrug resistant strains. Similar results have been reported by other workers. Our findings were similar to other investigators. Manjula et al (2005), Usha et al (2007), Garg et al (2007)

In our study MRSA were $53 \%$ and MRCoNS were 43\%. All gram positive cocci including MRSA, Enterococcus spp. and Coagulase negative Staphylococci were $100 \%$ sensitive to Vancomycin. Our findings are similar to Karthikeyan et al (2001), who reported that $66 \%$ of Staphylococcus aureus isolated from cases of neonatal sepsis were methicillin resistant. 
Table.1 Distribution of Microorganisms Isolated from Blood Cultures

\begin{tabular}{|l|l|l|}
\hline Organism & No. of isolates & Percentage \\
\hline Pseudomonas & 108 & $19 \%$ \\
\hline Staphylococcus aureus & 74 & $12.9 \%$ \\
\hline Klebsiella & 70 & $12.1 \%$ \\
\hline E coli & 63 & $11 \%$ \\
\hline CONS & 63 & $11 \%$ \\
\hline Enterobacter & 50 & $8.8 \%$ \\
\hline Citrobacter & 47 & $8.2 \%$ \\
\hline Acinetobacter & 40 & $7 \%$ \\
\hline Candida & 23 & $4 \%$ \\
\hline Enterococcus & 12 & $2.4 \%$ \\
\hline Salmonella & 11 & $1.9 \%$ \\
\hline Micrococci & 10 & $1.7 \%$ \\
\hline Total & 571 & \\
\hline
\end{tabular}

Table.2 Antibiotic Sensitivity Patterns of Gram-Positive Organisms in Percentage

\begin{tabular}{|l|l|l|l|}
\hline Antibiotics & CoNS $(n=63)$ & Staphylococcus aureus $(n=74)$ & Enterococci $(n=12)$ \\
\hline Vancomycin & $63(100 \%)$ & $74(100 \%)$ & $12(100 \%)$ \\
\hline Erythromycin & $36(57.1 \%)$ & $38(51.3 \%)$ & $5(41.6 \%)$ \\
\hline Clindamycin & $39(61.9 \%)$ & $43(58.1 \%)$ & $6(50 \%$ \\
\hline Cefotaxime & $43(68.2 \%)$ & $50(67.5 \%)$ & - \\
\hline Cefepime & $43(68.2 \%)$ & $58(78.3 \%)$ & - \\
\hline Ciprofloxacin & $54(85.74 \%)$ & $64(86.46 \%)$ & $12(100 \%)$ \\
\hline Teicoplanin & $59(93.6 \%)$ & $70(94.5 \%)$ & $12(100 \%)$ \\
\hline Gentamycin & $56(89 \%)$ & $65(87.8 \%)$ & $10(83.3 \%)$ \\
\hline Amoxycillin-clavulenate & $48(76.1 \%)$ & $58(78.3 \%)$ & $9(75 \%)$ \\
\hline Ampicillin & $31(49.2 \%)$ & $29(39.1 \%)$ & $1(8 \%)$ \\
\hline
\end{tabular}

Table.3 Antibiotic Sensitivity Patterns of Gram-Negative Organisms

\begin{tabular}{|c|c|c|c|c|c|c|c|}
\hline & $\begin{array}{l}\text { Psedomon } \\
\text { as } n=108\end{array}$ & $\begin{array}{l}\text { Citrobacter } \\
n=47\end{array}$ & $\begin{array}{l}\text { Klebsiella } \\
n=70\end{array}$ & $\begin{array}{l}\text { Acinetobact } \\
\text { er } n=40\end{array}$ & $\begin{array}{l}\text { E.coli } \\
n=63\end{array}$ & $\begin{array}{l}\text { Enterobacte } \\
r n=50\end{array}$ & $\begin{array}{l}\text { Salmonella } \\
n=11\end{array}$ \\
\hline Ampicillin & - & $13(27.6 \%)$ & $48(68.5 \%)$ & $27(67.5 \%)$ & $41(65 \%)$ & $25(50 \%)$ & $7(63.6 \%)$ \\
\hline Amikacin & $98(90.7 \%)$ & $43(91.4 \%)$ & $58(82.8 \%)$ & $36(90 \%)$ & $56(88.8 \%)$ & $42(84 \%)$ & $11(100 \%)$ \\
\hline Ceftazidime & $56(51.8 \%)$ & $26(55.3 \%)$ & $42(60 \%)$ & $22(55 \%)$ & $43(68.2 \%)$ & $30(60 \%)$ & $7(63.6 \%)$ \\
\hline Cefazolin & - & $31(65.9 \%)$ & $37(52.8 \%)$ & $23(57.5 \%)$ & $34(53.9 \%)$ & $32(64 \%)$ & $6(54.54 \%)$ \\
\hline Cefepime & - & $34(72.2 \%)$ & $37(52.8 \%)$ & - & $33(52.3 \%)$ & $30(60 \%)$ & $5(45.45 \%)$ \\
\hline Ciprofloxacin & - & $41(87.2 \%)$ & $58(82.8 \%)$ & $33(82.5 \%)$ & $54(85.7 \%)$ & $44(88 \%)$ & $11(100 \%)$ \\
\hline $\begin{array}{l}\text { Piperacillin- } \\
\text { tazobactam }\end{array}$ & $98(90.7 \%)$ & $40(85.1 \%)$ & $62(88.5 \%)$ & $35(87.5 \%)$ & $55(87.3 \%)$ & $45(90 \%)$ & $7(63.6 \%)$ \\
\hline Imepenem & $95(87.9 \%)$ & $42(89.3 \%)$ & $56(80 \%)$ & $37(92.5 \%)$ & $61(96.8 \%)$ & $50(100 \%)$ & $10(90.9 \%)$ \\
\hline Gentamycin & $95(87.9 \%)$ & $41(87.2 \%)$ & $64(91.4 \%)$ & $31(77.5 \%)$ & $58(92 \%)$ & $41(82 \%)$ & $9(81.8 \%)$ \\
\hline Cefotaxime & - & $34(72.2 \%)$ & $49(70 \%)$ & $31(77.5 \%)$ & $55(87.3 \%)$ & $35(70 \%)$ & $6(54.54 \%)$ \\
\hline Netilmycin & $97(89.8 \%)$ & $36(76.5 \%)$ & $54(77.1 \%)$ & $32(80 \%)$ & $57(90.4 \%)$ & $41(82 \%)$ & $8(72.72 \%)$ \\
\hline $\begin{array}{l}\text { Amoxycillin- } \\
\text { clavulenate }\end{array}$ & - & $38(80.8 \%)$ & $51(72.8 \%)$ & $36(90 \%)$ & $52(82.5 \%)$ & $42(84 \%)$ & $10(90.9 \%)$ \\
\hline Aztreonam & - & $26(55.3 \%)$ & $31(44.2 \%)$ & $23(57.5 \%)$ & $33(52.3 \%)$ & $34(68 \%)$ & $2(18.1 \%)$ \\
\hline Cotrimoxazol & - & $34(72.2 \%)$ & $51(72.8 \%)$ & $35(87.5 \%)$ & $54(85.7 \%)$ & $40(80 \%)$ & $7(63.6 \%)$ \\
\hline Ofloxacin & $89(82.4 \%)$ & - & - & - & - & - & - \\
\hline Tetracyclin & - & $26(55.3 \%)$ & $54(77.1 \%)$ & $33(82.5 \%)$ & $43(68.2 \%)$ & $35(70 \%)$ & $2(18.1 \%)$ \\
\hline
\end{tabular}


Table.4 Comparison of Various Studies from Various Places

\begin{tabular}{|l|l|l|}
\hline Study & Place & ESBL \\
\hline Baby padmini et al 2004 & Tamilnadu & $41 \%$ \\
\hline singhal et al 2005 & Gurgaon & $64 \%$ \\
\hline Sehgal et al 2007 & New delhi & $61.3 \%$ \\
\hline Shridhar Rao et al 2007 & Karnatak & $62.9 \%$ \\
\hline Sinha et al 2008 & Jaipur & $64.8 \%$ \\
\hline Dechen et al 2009 & Sikkim & $34 \%$ \\
\hline Chatergee 2010 & Chandigarh & $53.8 \%$ \\
\hline Akujobi 2010 & Nigeria & $57.6 \%$ \\
\hline Nasrin et al 2010 & Iran & $28.6 \%$ \\
\hline Sanjay et al 2010 & Mysore & $23.1 \%$ \\
\hline K Aruna et al 2012 & South Mumbai & $34.7 \%$ \\
\hline Amutha et al 2014 & Chennai & $67.3 \%$ \\
\hline Present study 2016 & Haryana & $21.1 \%$ \\
\hline
\end{tabular}

Table.5 Antibiotic Susceptibility Pattern of Esbl and Non Esbl Isolates

\begin{tabular}{|l|l|l|l|}
\hline Antibiotic & ESBL $\mathrm{n}=120$ & Non ESBL $\mathrm{n}=451$ & $\mathrm{P}$ value \\
\hline Ampicillin & 0 & $275(61 \%)$ & $<0.05$ \\
\hline Cotrimoxazol & $42(35 \%)$ & $311(69 \%)$ & $<0.05$ \\
\hline Tetracycline & $57(47.5 \%)$ & $302(67 \%)$ & $<0.05$ \\
\hline Ciprofloxacin & $64(53.4 \%)$ & $383(85 \%)$ & $<0.05$ \\
\hline Gentamycin & $78(65 \%)$ & $415(92 \%)$ & $<0.05$ \\
\hline Imepenem & $113(94 \%)$ & $451(100 \%)$ & $<0.05$ \\
\hline Piperacillin+Tazobactam & $79(65.8 \%)$ & $392(87 \%)$ & $<0.05$ \\
\hline Amoxycillin clavulenate & $53(44 \%)$ & $329(73 \%)$ & $<0.05$ \\
\hline
\end{tabular}

Isolation of MRSA was $31.25 \%$ as per Gandhi et al (2013), and it was 33\% according to Patel et al (2014). So screening for MRSA in every Staphylococcus aureus isolated will be of immense value for providing efficient patient care.

In our study $23 \%$ isolates were multi drug resistant (MDR). The infection caused by MDR organisms is more likely to prolong the hospital stay, increase the risk of death, and require treatment with more expensive antibiotics. Gene for ESBL, Amp C and MBL are often carried by plasmids, facilitating rapid spread between microorganism. Jain et al (2003) Presence of these enzymes in single isolates reduces the effectiveness of $\beta$ lactam and $\beta$ lactam inhibitor combinations, while Amp $\mathrm{C}$ and
MBL confer resistance to Carbepenems also. Often these enzymes are co-expressed in the same isolates. In almost all cases, antimicrobial therapy is initiated empirically before the results of blood culture are available. Keeping in mind the high mortality and morbidity associated with septicaemia, right choice of empiric therapy is of important. MRSA and MRCoNS indicated hospital acquired infection that needed strict infection control measures. The irrational use of broad spectrum $\beta$-lactam antibiotics has led to a marked increase in the incidence of ESBL in Gram negative organisms. ESBL were detected in $21.1 \%$ of Enterobacteriaceae. This is different from other studies from North India in which $61.5 \%$ were ESBL producers. Sehgal et al 
(2007). 6.4\% isolates showed Amp-C beta lactamases in those isolates. This is similar to observation made by Singhal et al (2005) which showed Amp-C beta lactamases in $8 \%$ isolates. The difference in percentage of isolation of ESBL compared to few other studies could be due to regional variations. Distinct regional variations have been detected in the incidence of ESBL producing isolates. Restricted use of Cephalosporins significantly reduced the incidence of ESBL. A significant difference $(\mathrm{P}$ value $<0.05)$ found between antibiotic susceptibility of ESBL and non ESBL strains. ESBL producing strains were more resistant to antimicrobial agents including Ampicillin, Cotrimoxazol, Tetracycline, Ciprofloxacin and Gentamycin. Imipenem was found to be most effective antibiotic against ESBL producing strain while in non ESBL strains Imipenem resistance was zero. ESBL producing strains were resistant to more antimicrobial agents than non ESBL producing strains. The highest rate of resistance in ESBL negative strains were seen against Ampicillin which was significantly lower than ESBL producing strains. None of the first line antibiotics used singly showed high susceptibility to all the Gram-negative bacilli, so a combination of two or more drugs is recommended to cover the broad range of possible pathogens which may be difficult to distinguish clinically. This may prevent the emergence of resistance as they may have additive or synergistic antimicrobial activity.

Simple hygienic measures, such as hand washing practices, the use of sterile equipment, cohorting (i.e. grouping patients with similar infections in the same location) of patients and attending staff for MRSA and ESBL can help prevent the further spread of these resistant strains. This study stresses that antimicrobial resistance is a global problem and emphasizes the need for surveillance and promotion of correct and restrictive antibiotic policies including specific antibiotic therapy after studying sensitivity pattern. This will halt the further spread of MRSA and ESBL and improve the prognosis in cases of sepsis.

In conclusion, these data provided much needed information on the prevalence of antimicrobial resistance amongst pathogens causing blood stream infections our area. The rise in antibiotic resistance in blood isolates emphasises the importance of sound hospital infection control, rational prescribing policies, and the need for new antimicrobial drugs and vaccines. Resistance to broad spectrum $\beta$ lactams (ESBL, AmpC and $\mathrm{MBL}$ ) can result in treatment failure. Therefore it is recommended that any ESBL producing organism according to national committee for clinical laboratory standards (NCCLS) can be reported as resistant to all extended spectrum $\beta$ lactam antibiotics regardless of susceptibility test results. Our results seem helpful in providing Useful guidelines for choosing an effective antibiotic in cases of septicaemia and for choosing salvage therapy against hospital resistant strain.

\section{References}

Akujobi CN, Ewuru Chika P Detection of extended spectrum beta lactamases in Gram negative Bacilli from clinical specimen in a teaching hospital in South Eastern Nigeria Nigerian Medical Journal 2010 ;51: 141-46

Amutha Chelliah, Thyagarajan Ravinder, RadhikaKatragadda, K.V. Leela, R. NarayanaBabu Isolation of of MRSA, ESBL and AmpC - $\beta$-lactamases from Neonatal Sepsis at a Tertiary Care Hospital Journal of Clinical and Diagnostic Research. 2014;8:24-27

Anantham S, Subha A, Cefoxitin Resistance mediated by loss of a porin in clinical 
strains of Klebsiella pneumoniae and $\mathrm{E}$ coli. Indian Journal Medical Microbiology 2005;23:20-3

Araya Gebreyesus Wasihun, Letemichael Negash Wlekidan, Bacteriological profile and antimicrobial susceptibility patterns of blood culture isolates among febrile patients in Mekelle Hospital, Northern Ethiopia. SpringerPlus 2015; 4:314

Atul Garg, S Anupurba, Jaya Garg, RK Goya, MR Sen Bacteriological Profile and Antimicrobial Resistance of Blood Culture Isolates from a University Hospital Journal, Indian Academy of Clinical Medicine 2007; 8(2): 139-43

Babypadmini S, Appalaraju B extanded spectrum Beta lactamases in urinary isolates of $\mathrm{E}$ coli and klebsiella pneumoniae prevalence and susceptibility pattern in a tertiary care hospital. Indian Journal Medical Microbiology 2004;22:172-4

Bailey and Scott's Diagnostic microbiology $12^{\text {th }}$ edition Betty A Forbes, Daniel F Sahm, Alice S Weissfeld Blood stream Infections 2007 pp 785-88

Black J A, Thomson KS, Buynak JD. Evaluation of beta lactamases inhibitor in disc test for detection of plasmid mediated Amp C beta lactamases in well characterized clinical strains of Klebsiella spp. Journal of Clinical Microbiology 2005;43:4168-71

Broekema NM, Van TT, Monson TA, Marshall SA, Warshauer DM. Comparison of cefoxitin and oxacillin disc diffusion methods for detection of Mec A mediated resistance in Staphylococcus aureus in a large scale study. Journal Clinical Microbiology 2009; 47(1): 217-19.

Chatterjee SS, Karmacharya K High prevalence of co- expression of newer Beta lactamases (ESBL, Amp C beta lactamases and metallo beta lactamases) in gram negative bacilli Indian Journal of Medical Microbiology 2010; 28;267-68

Clinical and Laboratory Standards Institute (CLSI) (2014) Performance standards for antimicrobial susceptibility testing; twenty-fourth informational supplement, CLSI document M100S24. Wayne and Pennsylvania

Dechen C Tsering, Shyamasree Das Extended spectrum beta lactamases detection in gram negative bacilli of nosocomial origin Journal of Global Infectious Diseases 2009;1:87-92

Gandhi S, Ranjan KP, Ranjan N, Sapre N, Masani M. Incidence of neonatal sepsis in tertiary care hospital: an overview International Journal of Medical Science and Public Health. 2013; 2(3): 548- 52

Gupta V, Singla N, Chander J, Detection of ESBLs using third and fourth generation cephalosporins in double disc synergy test. Indian Journal of Medical Research 2007;126:486-7

Jain A, Roy I Gupta MK, prevalence of extended spectrum beta lactamases producing gram negative bacteria in septicaemic neonates in a tertiary care hospital Indian Journal of Medical Microbiology 2003;52: 421-5

Jarlier V, Nicolas MH, Fournier G, Philippon A. Extended broad-spectrum $\beta$-lactamasesconferring transferable resistance to newer $\beta$-lactam agents in Enterobacteriaceae:Hospital prevalence and susceptibility patterns. Review of Infectious disease 1988; 10:867-78

Aruna, K. T. Mobashshera prevalence of extended spectrum beta lactamases production among uropathogens in south Mumbai and its antibiogram pattern EXCLI Journal 2012;11:363372

Kalpesh Gohel, Amit Jojera, Shailesh Soni, Sishir Gang, Ravindra Sabnis, and 
Mahesh Desai Bacteriological Profile and Drug Resistance Patterns of Blood Culture Isolates in a Tertiary Care Nephrourology Teaching Institute Biological Medical Research International 2014;10

Karthikeyan G, Premkumar K. Neonatal sepsis-Staphylococcus aureus as predominant pathogen Indian Jounal of Pediatrics. 2001;68(8):715-17.

Kumar MS, Lakshmi V, occurrence of extended spectrum beta lactamases among enterobacteriaceae spp.isolated at tertiary care institute. Indian Journal of Medical Microbiology 2006;24:20811

Lal P, Kapil A, Das BK, Sood S, Occurence of Tem and SHV gene in extended spectrum beta lactamases producing klebsiella sp. isolated from a teriary care hospital Indian Journal of Medical Research 2007;125:173-8

Magiorakos AP, Srinivasan A, Carey RB, Carmeli Y, Falagas ME,Giske CG, et al Multidrug-resistant, extensively drugresistant and pandrug-resistant bacteria: an international expert proposal for interim standard definitions for acquired resistance. European society of Clinical Microbiology and Infectious diseases 2012;18: 268281

Manjula Mehta, Priya Dutta, Varsha Gupta Antimicrobial Susceptibility Pattern of Blood Isolates from a Teaching Hospital in North India, Japanese Journal of Infectious Disease, 2005;58:174-176

Marchiaro P, Mussi MA, Ballerini V, Pasteran F, sensitive EDTA based microbiological assay for detection of mettalo-beta- lactamases in non fermentative gram negative bacteria. Journal of Clinical Microbiology 2005;43:5648-52

Nasrin Shayanfar, Mitra Rezaei, Mehdi Ahmadi, Fahime Ehsanipour
Evaluation of Extended Spectrum Betalactamase (ESBL) Positive Strains of Klebsiella pneumoniae And Escherichia coli in Bacterial Cultures Iranian Journal of pathology 2010;5:34-39

Patel D, Nimbalkar A, Sethi A, Kungwani A, Nimbalkar S. Blood culture isolates in neonatal sepsis and their sensitivity in Anand district of India. Indian Journal of Pediatric. 2014;81:785-90

Sanjay K R, M. N. Nagendra Prasad and G. S. Vijaykumar A study on isolation and detection of drug resistance gram negative bacilli with special importance to post operative wound infection Journal of Microbiology and Antimicrobials 2010;2(6), ;68-75,

Sehgel R, Gaind R, Chellani H, Agarwal P. Extended-spectrum $\quad \beta \quad$ lactamaseproducing gram-negative bacteria: clinical profile and outcome in a neonatal intensive care unit. Annal Tropical Paediatric. 2007;27(1):45-54.

Shridhar Rao, KG Basavarajappa, Detection of extended spectrum beta lactamases from clinical isolates in Davangere Indian Journal of Pathology and Microbiology 2008;51(4):497-99

Singhal S, Thakur T, Khan S, Upadhyay DJ, Chugh S, Gaind R et al. Evaluation of methods for Amp C beta lactamases in gram negative clinical isolates from tertiary care hospitals. Indian Journal Medical Microbiology. 2005; 23(2): $120-24$

Sinha P, Sharma R, Rishi S prevalence of extended spectrum beta lactamases and Amp C beta lactamases producers among E coli isolates in a tertiary care hospital in Jaipur Indian J of Pathology and Microbiology 2008;51;367-69

Sumita Rajeevan, Syed Mustaq Ahmad and P.T.Jasmin Study of prevalence and antimicrobial susceptibility pattern in blood isolates from a tertiary care 
hospital in North Kerala, India International Journal of Current Microbiology Applied Science 2014;3(4): 655-662

Usha Arora, Pushpa Devi Bacterial Profile of Blood Stream Infections and Antibiotic Resistance Pattern of Isolates $j k$ science 2007; 9: 4

Vanitha Rani N, Kannan Gopal, Venkata Narendra M, Vishwakanath D, V R D Nagesh, Yogitha M, A Retrospective study on blood stream infection and antibiotic susceptibility pattern in a tertiary care teaching hospital International Journal of Pharmacy and Pharmaceutical Sciences2012; 4:1

VinodKumar C S, Kalappanavar NK, Umakanth Patil, Basavarajappa K Change in spectrum of microbial aetiology in relation to gestational age and birth weight and emergence of ESBL in tertiary neonatal intensive care units. International Journal of Biological Medical Research 2011; 2(3): 727-734

\section{How to cite this article:}

Ruby Naz, Mohammad Khalid Farooqui, Ruchi Girotra and A. K. Malik. 2016. Bacteriological Profile of Septicaemia and Extended Spectrum Beta Lactamases Production in Multi Drug Resistant Strains. Int.J.Curr.Microbiol.App.Sci.5(2): 598-607. doi: http://dx.doi.org/10.20546/ijcmas.2016.502.067 